\title{
Estimates of THE Bounds of
}

\section{$\pi(x)$ AND $\pi\left((x+1)^{2}\right)-\pi\left(x^{2}\right)$}

\section{CONNOR PAUL WILSON}

We show the following bounds on the prime counting function $\pi(x)$ using principles from analytic number theory, giving an estimate

$$
\log 2 \leq \liminf _{x \rightarrow \infty} \frac{\pi(x)}{x / \log x} \leq \limsup _{x \rightarrow \infty} \frac{\pi(x)}{x / \log x} \leq 2 \log 2
$$

We also conjecture about the bounding of $\pi\left((x+1)^{2}\right)-\pi\left(x^{2}\right)$, as is relevant to Legendre's conjecture about the number of primes in the aforementioned interval such that:

$$
\begin{gathered}
\left\lfloor\frac{1}{2}\left(\frac{(x+1)^{2}}{\log (x+1)}-\frac{x^{2}}{\log x}\right)-\frac{(\log x)^{2}}{\log (\log x)}\right\rfloor \leq \pi\left((x+1)^{2}\right)-\pi\left(x^{2}\right) \leq \\
\left\lfloor\frac{1}{2}\left(\frac{(x+1)^{2}}{\log (x+1)}-\frac{x^{2}}{\log x}\right)-\log ^{2} x \log \log x\right\rfloor
\end{gathered}
$$

\section{Introduction}

Recall the definition:

$$
\pi(x):=\sum_{\substack{p \leq x \\ p \text { prime }}} 1
$$

Contact: Connor Paul Wilson <dpoae@umich.edu> 
and let us define the following:

$$
\begin{gathered}
\Theta(x):=\sum_{\substack{p \leq x \\
p \text { prime }}} \log p, \\
\Psi(x):=\sum_{1 \leq n \leq x} \Lambda(n)=\sum_{\substack{p^{m} \leq x \\
m \geq 1 \\
p \text { prime }}} \log p, \\
\Lambda(n)= \begin{cases}\log p & \text { if } n=p^{k} \text { for some prime } p \text { and integer } k \geq 1 \\
0 & \text { otherwise. }\end{cases}
\end{gathered}
$$

The following are simple statements from real analysis that are required for rigorousness' sake: let $\left\{x_{n}\right\}$ be a sequence of real numbers and $L$ be a real number with the following two properties: $\forall \epsilon>0, \exists N$ such that $x_{n}<L+\epsilon, \forall n \geq N$. $\forall \epsilon>0$ $\wedge N \geq 1, \exists n \geq N$ with $x_{n}>L-\epsilon$. We thus define $L$ as:

$$
\limsup _{n \rightarrow \infty} x_{n}=L
$$

Thus on the contrary we must have:

$$
\liminf _{n \rightarrow \infty} x_{n}=-\limsup -x_{n}
$$

\section{Necessary Preliminary Results}

Theorem 2.1. For all $\alpha \in(0,1)$, and all $x \geq x_{0}$ :

$$
\frac{\Theta(x)}{\log (x)} \leq \frac{\Psi(x)}{\log (x)} \leq \pi(x) \leq \frac{\Theta(x)}{\alpha \log (x)}+x^{\alpha}
$$

Proof. Clearly $\Theta(x) \leq \Psi(x)$, such that

$$
\limsup _{x \rightarrow \infty} \frac{\Psi(x)}{x} \geq \limsup _{x \rightarrow \infty} \frac{\Theta(x)}{x}
$$

Also, if $p$ is a prime and $p^{m} \leq x<p^{m+1}$, then $\log p$ occurs in the sum for $\Psi(x)$ exactly $m$ times. [1] 


$$
\begin{aligned}
\Psi(x) & =\sum_{\substack{p^{m} \leq x \\
p \text { prime } \\
m \geq 1}} \log p \\
& =\sum_{\substack{p \leq x \\
p \text { prime }}}\left[\frac{\log x}{\log p}\right] \log p \\
& \leq \sum_{\substack{p \leq x \\
p \text { prime }}} \log x \\
& =\pi(x) \log x
\end{aligned}
$$

$$
\limsup _{x \rightarrow \infty} \frac{\Psi(x)}{x} \leq \limsup _{x \rightarrow \infty} \frac{\pi(x)}{x / \log x}
$$

Now fix $\alpha \in(0,1)$. Given $x>1$,

$$
\Theta(x)=\sum_{\substack{p \leq x \\ p \text { prime }}} \log p \geq \sum_{\substack{x^{\alpha}<p \leq x \\ p \text { prime }}} \log p
$$

It is clear that all $p$ from the second sum satisfy: $\log p>\alpha \log x$.

$$
\begin{aligned}
\Theta(x)> & \alpha \log x \sum_{\substack{x^{\alpha}<p \leq x \\
p \text { prime }}} 1 \\
& =\alpha \log x\left(\pi(x)-\pi\left(x^{\alpha}\right)\right) \\
& >\alpha \log x\left(\pi(x)-x^{\alpha}\right)
\end{aligned}
$$

$\ni$

$$
\frac{\Theta(x)}{x}>\frac{\alpha \pi(x)}{x / \log x}-\frac{\alpha \log x}{x^{1-\alpha}}
$$

$\forall \alpha \in(0,1)$ we have:

$$
\lim _{x \rightarrow \infty} \frac{\alpha \log x}{x^{1-\alpha}}=0
$$


4 - Connor Paul Wilson

Combining these we get:

$$
\limsup _{x \rightarrow \infty} \frac{\Theta(x)}{x} \geq \limsup _{x \rightarrow \infty} \frac{\alpha \pi(x)}{x / \log x}
$$

Once again, since our statement is true $\forall \alpha \in(0,1)$,

$$
\limsup _{x \rightarrow \infty} \frac{\Theta(x)}{x} \geq \limsup _{x \rightarrow \infty} \frac{\pi(x)}{x / \log x}
$$

Similarly:

$$
\begin{gathered}
\liminf _{x \rightarrow \infty} \frac{\Psi(x)}{x} \geq \liminf _{x \rightarrow \infty} \frac{\Theta(x)}{x} \\
\liminf _{x \rightarrow \infty} \frac{\pi(x)}{x / \log x} \geq \liminf _{x \rightarrow \infty} \frac{\Psi(x)}{x}
\end{gathered}
$$

Once again, we apply the same method:

$$
\liminf _{x \rightarrow \infty} \frac{\Theta(x)}{x} \geq \liminf _{x \rightarrow \infty} \frac{\pi(x)}{x / \log x}
$$

and have thus proven Theorem 2.1.

\section{Main Result}

Theorem 3.1. We have:

$$
\log 2 \leq \liminf _{x \rightarrow \infty} \frac{\pi(x)}{x / \log x} \leq \limsup _{x \rightarrow \infty} \frac{\pi(x)}{x / \log x} \leq 2 \log 2
$$

Proof. First the lower bound. Take:

$$
S(x):=\sum_{1 \leq n \leq x} \log n-2 \sum_{1 \leq n \leq x / 2} \log n .
$$


Then,

$$
\begin{aligned}
\sum_{\substack{1 \leq d \leq n \\
d \mid n}} \Lambda(d) & =\sum_{\substack{p^{r} \mid d \\
\text { aprime } \\
\text { p.rime }}} \log p \\
& =\sum_{i=1}^{l} \sum_{r=1}^{e_{i}} \log p_{i} \quad \text { where } n=p_{1}^{e_{1}} \cdots p_{l}^{e_{l}} \\
& =\sum_{i=1}^{l} e_{i} \log p_{i} \\
& =\log n
\end{aligned}
$$

$$
S(x)=\sum_{1 \leq n \leq x} \sum_{d \mid n} \Lambda(d)-2 \sum_{1 \leq n \leq x / 2} \sum_{d \mid n} \Lambda(d)
$$

Clearly $\{d, 2 d, \ldots, q d\}$ is the set of $n$ satisfying $1 \leq n \leq x$ and $d \mid n$ (we can see this easily by writing $x=r+q d$ with $0 \leq r<d$ ).

$$
\begin{aligned}
S(x) & =\sum_{1 \leq d \leq x} \Lambda(d)\left[\frac{x}{d}\right]-2 \sum_{1 \leq d \leq x / 2} \Lambda(d)\left[\frac{x}{2 d}\right] \\
& =\sum_{1 \leq d \leq x / 2} \Lambda(d)\left(\left[\frac{x}{d}\right]-2\left[\frac{x}{2 d}\right]\right)+\sum_{(x / 2)<d \leq x} \Lambda(d)\left[\frac{x}{d}\right] \\
& \leq \sum_{1 \leq d \leq x / 2} \Lambda(d)+\sum_{(x / 2)<d \leq x} \Lambda(d) \\
& =\Psi(x) .
\end{aligned}
$$

So,

$$
\frac{\Psi(x)}{x} \geq \frac{S(x)}{x}=\frac{1}{x} \sum_{1 \leq n \leq x} \log n-\frac{2}{x} \sum_{1 \leq n \leq x / 2} \log n .
$$

$\log t$ is increasing,

$$
\begin{aligned}
& \int_{1}^{x+1} \log t d t \geq \sum_{1 \leq n \leq x} \log n \\
& \int_{1}^{[x]} \log t d t \leq \sum_{1 \leq n \leq x} \log n .
\end{aligned}
$$


6 - Connor Paul Wilson

Actually, assuming $x \in \mathbb{Z}^{+}$,

$$
\begin{aligned}
\frac{S(x)}{x} & \geq \frac{1}{x} \int_{1}^{x} \log t d t-\frac{2}{x} \int_{1}^{(x / 2)+1} \log t d t \\
& =\frac{1}{x}(x \log x-x+1)-\frac{2}{x}\left(\frac{x+2}{2} \log \left(\frac{x+2}{2}\right)-\frac{x+2}{2}+1\right) \\
& =\log x+\frac{1}{x}-\frac{x+2}{x} \log (x+2)+\frac{x+2}{x} \log 2 \\
& >\log \left(\frac{x}{x+2}\right)-\frac{2}{x} \log (x+2)+\log 2
\end{aligned}
$$

Using Theorem 2.1, we get:

$$
\begin{aligned}
\liminf _{x \rightarrow \infty} \frac{\pi(x)}{x / \log x} & =\liminf _{x \rightarrow \infty} \frac{\Psi(x)}{x} \\
& \geq \liminf _{x \rightarrow \infty} \frac{S(x)}{x} \\
& >\lim _{x \rightarrow \infty} \log (x /(x+2))-\frac{2}{x} \log (x+2)+\log 2 \\
& =\log 2
\end{aligned}
$$

To complete the proof, we will need some auxiliary results taken from Murty's Analytic Number Theory [1] in the form of three lemmas:

\section{Lemma 3.2.}

$$
\operatorname{ord}_{p}(m !)=\sum_{r \geq 1}\left[\frac{m}{p^{r}}\right], \forall m \in \mathbb{Z}^{+}, \text {prime } p
$$

Proof. Fix an exponent $r$. The positive integers no larger than $m$ that are multiples of $p^{r}$ are

$$
p^{r}, 2 p^{r}, \ldots,\left[\frac{m}{p^{r}}\right] p^{r}
$$

and those that are multiples of $p^{r+1}$ are

$$
p^{r+1}, 2 p^{r+1}, \ldots,\left[\frac{m}{p^{r+1}}\right] p^{r+1}
$$


Estimates of the Bounds of $\pi(x)$ and $\pi\left((x+1)^{2}\right)-\pi\left(x^{2}\right) \quad \cdot 7$

Thus there are precisely $\left[m / p^{r}\right]-\left[m / p^{r+1}\right]$ positive integers $n \leq m$ with $\operatorname{ord}_{p}(n)=r$.

$$
\begin{aligned}
\operatorname{ord}_{p}(m !) & =\sum_{n=1}^{m} \operatorname{ord}_{p}(n) \\
& =\sum_{r \geq 1} \sum_{\substack{1 \leq n \leq m \\
\operatorname{ord}_{p}(n)=r}} r \\
& =\sum_{r \geq 1} r\left(\left[m / p^{r}\right]-\left[m / p^{r+1}\right]\right) \\
& =\sum_{r \geq 1} r\left[m / p^{r}\right]-\sum_{r \geq 1} r\left[m / p^{r+1}\right] \\
& =\sum_{r \geq 1} r\left[m / p^{r}\right]-\sum_{r \geq 1}(r-1)\left[m / p^{r}\right] \\
& =\sum_{r \geq 1}\left[\frac{m}{p^{r}}\right]
\end{aligned}
$$

Lemma 3.3. $\forall n \in \mathbb{Z}^{+}$,

$$
\frac{2^{2 n}}{2 \sqrt{n}}<\left(\begin{array}{c}
2 n \\
n
\end{array}\right)<\frac{2^{2 n}}{\sqrt{2 n+1}}
$$

Proof.

$$
\begin{aligned}
P_{n} & :=\prod_{i \leq n} \frac{(2 i-1)}{(2 i)} \\
& =\frac{(2 n) !}{2^{2 n}(n !)^{2}} \\
& =\left(\begin{array}{c}
2 n \\
2
\end{array}\right) \frac{1}{2^{2 n}}
\end{aligned}
$$

Since:

$$
\frac{(2 i-1)(2 i+1)}{(2 i)^{2}}<1
$$

for all $i \geq 1$.

$$
1>(2 n+1) P_{n}^{2}
$$


8 - Connor Paul Wilson

giving the upper bound. For the lower bound:

$$
1-\frac{1}{(2 i-1)^{2}}<1
$$

$\forall i \geq 1$, such that

$$
\begin{gathered}
1>\prod_{i=2}^{n}\left(1-\frac{1}{(2 i-1)^{2}}\right) \\
=\prod_{i=2}^{n} \frac{(2 i-1)^{2}-1}{(2 i-1)^{2}} \\
=\prod_{i=2}^{n} \frac{(2 i-2)(2 i)}{(2 i-1)^{2}} \\
=\frac{1}{4 n P_{n}^{2}}
\end{gathered}
$$

yielding our lemma.

Lemma 3.4. $\forall n \in \mathbb{Z}^{+}$,

$$
\Theta(n)<2 n \log 2
$$

Proof. By Lemma 3.3,

$$
\begin{aligned}
\log \left(\left(\begin{array}{c}
2 n \\
n
\end{array}\right) \frac{1}{2}\right) & =\log \left(\left(\begin{array}{c}
2 n \\
n
\end{array}\right)\right)-\log 2 \\
& <2 n \log 2-\frac{1}{2} \log (2 n+1)-\log 2 \\
& =(2 n-1) \log 2-\frac{1}{2} \log (2 n+1)
\end{aligned}
$$

since

$$
\left(\begin{array}{c}
2 n \\
n
\end{array}\right) \frac{1}{2}=\frac{(2 n) !}{(n !)^{2}} \frac{n}{2 n}=\frac{(2 n-1) !}{n !(n-1) !}=\left(\begin{array}{c}
2 n-1 \\
n-1
\end{array}\right) .
$$


by Lemma 3.2:

$$
\begin{aligned}
\log \left(\left(\begin{array}{c}
2 n \\
n
\end{array}\right) \frac{1}{2}\right) & =\log \left(\begin{array}{c}
2 n-1 \\
n-1
\end{array}\right) \\
& =\sum_{p \text { prime }} \operatorname{ord}_{p}((2 n-1) !) \log p-\sum_{p \text { prime }} \operatorname{ord}_{p}((n-1) !) \log p-\sum_{p \text { prime }} \operatorname{ord}_{p}(n !) \log p \\
& =\sum_{p \text { prime }} \log p \sum_{r \geq 1}\left[(2 n-1) / p^{r}\right]-\left[n / p^{r}\right] \\
& \geq \sum_{\substack{p \text { prime } \\
n<p \leq 2 n-1}} \log p \\
& =\Theta(2 n-1)-\Theta(n)
\end{aligned}
$$

Where

$$
\Theta(2 n-1)-\Theta(n)<(2 n-1) \log 2-\frac{1}{2} \log (2 n+1)
$$

We now proceed by induction. Proceeding from the trivialities, suppose $m>2$ and the lemma is true for $n<m, n, m \in \mathbb{N}$. If $m$ is odd, then $m=2 n-1$ for some integer $n \geq 2$ since $m>2$. Thus by induction,

$$
\begin{aligned}
\Theta(m) & =\Theta(2 n-1)<\Theta(n)+(2 n-1) \log 2-\frac{1}{2} \log (2 n+1) \\
& <2 n \log 2+(2 n-1) \log 2-\frac{1}{2} \log (2 n) \\
& =(4 n-1) \log 2-\frac{1}{2} \log (2 n) \\
& \leq(4 n-2) \log 2 \quad(\text { since } n \geq 2) \\
& =2 m \log 2
\end{aligned}
$$

If $m$ is even, then $m=2 n$ for some integer $n$ with $m>n \geq 2$ and $m$ is composite. Clearly $\Theta(m)=\Theta(m-1)$ and we know:

$$
\Theta(m)=\Theta(m-1)<2(m-1) \log 2<2 m \log 2
$$


Lemma 3.4 gives

$$
2 \log 2 \geq \lim \sup _{x \rightarrow \infty} \frac{\Theta(x)}{x}
$$

The desired lower bound follows from Theorem 2.1

\section{On Primes in the Gaps between Squares}

The following is relatively aleatory compared to the previous workings, but it is worth mentioning considering the importance of the statement.

By Hassani [2], we have

$$
\begin{gathered}
\left\lfloor\frac{1}{2}\left(\frac{(x+1)^{2}}{\log (x+1)}-\frac{x^{2}}{\log x}\right)-\frac{\log ^{2} x}{\log \log x}\right\rfloor \leq \pi\left((x+1)^{2}\right)-\pi\left(x^{2}\right) \\
\quad \frac{1}{2}\left(\frac{x^{2}}{\log n}-\frac{3^{2}}{\log 3}\right)-\sum_{j=3}^{x-1} \frac{\log ^{2} x}{\log \log k}<\pi\left(x^{2}\right)-\pi\left(3^{2}\right)
\end{gathered}
$$

And thus:

$$
\begin{aligned}
& \sum_{j=3}^{x-1}\left\lfloor\frac{1}{2}\left(\frac{(j+1)^{2}}{\log (j+1)}-\frac{j^{2}}{\log j}\right)-\frac{\log ^{2} j}{\log \log j}\right\rfloor<\sum_{j=3}^{n-1} \pi\left((j+1)^{2}\right)-\pi\left(j^{2}\right) \\
& \left\lfloor\frac{1}{2}\left(\frac{(x+1)^{2}}{\log (x+1)}-\frac{x^{2}}{\log x}\right)-\frac{(\log x)^{2}}{\log (\log x)}\right\rfloor \leq \pi\left((x+1)^{2}\right)-\pi\left(x^{2}\right) .
\end{aligned}
$$

And by the prime number theorem, which gives us the asymptotic estimate for some

$$
F(x):=\pi\left((x+1)^{2}\right)-\pi\left(x^{2}\right) \sim \frac{1}{2}\left(\frac{(x+1)^{2}}{\log (x+1)}-\frac{x^{2}}{\log x}\right)
$$


We propose:

$$
\pi\left((x+1)^{2}\right)-\pi\left(x^{2}\right) \leq\left\lfloor\frac{1}{2}\left(\frac{(x+1)^{2}}{\log (x+1)}-\frac{x^{2}}{\log x}\right)+\log ^{2} x \log \log x\right\rfloor
$$

by the same method.

\section{References}

1. R. Ram Murty, Problems in Analytic Number Theory, Second Edition - Graduate Texts in Mathematics, Springer, 2001.

2. M. Hassani, Counting primes in the interval $\left(n^{2},(n+1)^{2}\right)$, AMS, 1997 . 